\title{
Operational durability of reinforced concrete structures
}

\author{
Mikhail Berlinov ${ }^{1}$, Marina Berlinova ${ }^{1, *}$, Artem Grigorjan ${ }^{1}$ \\ ${ }^{1}$ Moscow State University of Civil Engineering, Yaroslavskoe shosse, 26, Moscow, 129337, Russia
}

\begin{abstract}
The resistance properties of reinforced concrete and reinforcement, required for modeling the degradation and calculations of the durability of reinforced concrete elements under the conditions of various types of corrosion damage, based on analytical and experimental data are substantiated. A probabilistic model has been developed for predicting and estimating the durability and reliability of reinforced concrete elements, taking into account the statistical variability of the calculation parameters and the kinetics of degradation processes during long periods of operation. Methods are proposed for calculating and predicting the durability of reinforced concrete elements based on deterministic probabilistic models of degradation of structural elements operating under aggressive environmental influences.
\end{abstract}

\section{Introduction}

In conditions of long-term operation of reinforced concrete structures in aggressive environments, it is necessary to evaluate their stress-strain state as a result of corrosion damage, to identify their residual life, given the reduction in the cross-sectional area of concrete and reinforcement $[1,2]$.

Corrosive damage to reinforced concrete elements can affect the strength of the material, change the design calculations, redistribute forces in the construction sections and disrupt the joint work of concrete with reinforcement, as well as lead to other consequences that reduce the deformation characteristics of reinforced concrete [3, 4]. The most unfavorable result of the development of the corrosion process in reinforced concrete structures is the reduction of their bearing capacity and their suitability for operation, which leads to non-compliance with safety requirements and the limiting state at design loads [5].

Corrosion of reinforcing steel is one of the most common damage to reinforced concrete. Initially, the corrosion of the reinforcement is caused by the violation of operating conditions, the aggressive influence of the external environment, the reduction of the protective characteristics of reinforced concrete, design errors and manufacturing defects, and the operating life of the structure can be significantly reduced [6].

Corrosion effects of various types of structures are exposed to bridges, parking lots and swimming pools, and other structures that are used in aggressive conditions. The study of

\footnotetext{
${ }^{*}$ Corresponding author: marina.tvor@mail.ru
} 
corrosion processes also contributes to the solution of the problems of corrosion of concrete and reinforcement in general.

In general, consideration of the problem of reliability of reinforced concrete structures and constructions taking into account corrosion damage is implemented by creating methods for predicting and assessing durability. When analyzing the durability properties of reinforced concrete structures, it is necessary to determine the following features of this problematic: the first is the probabilistic nature of non-force and power influences, their interrelation and complexity; the second is the instability of the technical qualities of the constructions and materials; and finally, the third - the influence of the time factor on the properties of materials and the characterological features of the effects.

\section{Results section}

Damage of a concrete element not loaded by external forces caused by unilateral influence of a chemical aggressor of constant characteristics (composition, intensity, temperature, barometric pressure) in conditions of a stable compressed humidity of the environment and concrete, considered to be elements obeying the non-linearly generalized Golberg-Waage law with entropic aging assessment of concrete destruction:

$$
\frac{d \Delta \delta_{0}\left(t, t_{0}\right)}{d t}=-\alpha\left[\Delta \delta_{0}\left(t, t_{0}\right)\right]^{m}
$$

where: $\Delta \delta\left(t, t_{0}\right)=\frac{\delta_{c r}\left(t_{0}\right)-\delta\left(t, t_{0}\right)}{\delta_{c r}\left(t_{0}\right)}=1-\frac{\Delta \delta\left(t, t_{0}\right)}{\delta_{c r}\left(t_{0}\right)}$, - the depth of damages, by the time t, $\delta\left(t, t_{0}\right)$ - current damage depth; $\delta_{c r}\left(t_{0}\right)$ - critical, in particular, the maximum depth in case of decreasing type of damage, depending on $\sigma / R ; \Delta \delta\left(t, t_{0}\right)$ - current relative damage deficit;

- $t$, $t_{0}$ - start time and current monitoring time; $\alpha, m$ - parameters of damage kinetics, determined experimentally for each variant of the combination of concrete and aggressor, depending on the level of the stress state $\sigma / R$.

$\alpha$ и $m$-some empirical values ( $\alpha$ - characterizes the rate of slowing down the process, $m$ - represents the type of corrosion model).

Solution (1) is possible in the form:

$$
\delta\left(t, t_{0}\right)=f_{m}(\alpha, m, t) \delta_{c r}\left(t_{0}\right)
$$

where:

$$
f_{m}=1-\left\{\left[\Delta \delta\left(t_{0}, t_{0}\right)\right]^{[(-m)+1]}+\alpha[(-m)+1]\left(t-t_{0}\right)\right\}^{\left.\frac{1}{[(-m)+1}\right]}
$$

Moreover, for each combination of concretes of different nominations and different concentrations such as corrosive aggressors (as well as temperatures, etc.), their numerical values of the specified parameters are calculated.

Thus, for each level of the stress state, there are numerical values of its parameters $\delta_{c r}$, $m, \alpha$ which can be taken from experimental data and correspond to the $\mathrm{n}$-th combination of concrete and aggressor, which gives a sufficient set of information to assess the kinetics of damage. 
The mechanical characteristics of damaged concrete as a result of corrosion are taken as the multiplication of their initial values on the damage function in the form of a power series and are found from the fixed values of the $K_{C}$, depending on the distance $Z$ measured from the contact surface of the corrosive medium and concrete:

$$
K_{C}=\left[1-\left(\frac{p}{\delta}\right)^{2}\right]+\frac{2 p}{\delta^{2}} z-\frac{1}{\delta^{2}} z^{2}
$$

here: $\mathrm{p}$ and $\delta$, respectively, the height of the intact and damaged layers of the concrete section.

With the help of (4) one can calculate the amount of the remaining force resistance, and take into account the amount lost due to the corrosion damage of the force resistance in relation to the evaluation of strength and deformability.

The reduction in the cross-sectional area of reinforcement, as an aggregate design factor, is calculated using the following formula:

$$
\begin{gathered}
A_{S}^{*}=\frac{\pi(D-2 \delta)^{2}}{4} \\
\delta=\frac{k}{\sqrt{b}} t^{n}
\end{gathered}
$$

$k$ and $n$-empirical coefficients, $b$ - the value of the protective layer, mm.

Thus, the calculated values of the cross-sectional area of the working reinforcement, as well as the mechanical characteristics of concrete under corrosion damage conditions, are not constant along the cross-section height. In the area located near the zone of maximum corrosion impact, their values are minimal and gradually increase, reaching maximum values in the zone of stabilization of the degradation process.

The probability that the resistance of reinforced concrete constructions will be less than the load, and the probability of completion of the service life must be less than the permissible probability of destruction:

$$
P_{v}(t)=P_{r s}\left[\left\{R\left(t_{e}\right)-Q\left(t_{e}\right)\right\}_{t_{e}}=S \prec 0\right] \prec P_{v, \text { max }}(t)=\int_{0}^{x} F_{R}(x) f_{Q}(x) d x
$$

where: $P_{v}(t)$ is the probability of constructions failure during $-t_{e}$ of a given (assigned) service life; $P_{r s}$ - the probability of the event; $P_{v, \max }(t)$ is the maximum allowable probability of destruction, the latter can be defined as the level of safety (reliability) of actually operated constructions; $F_{R}$ is the probability distribution function of the strength $\mathrm{R} ; f_{Q}$ is the area of the probability distribution of the magnitude of the force $Q$ (Fig.1). 


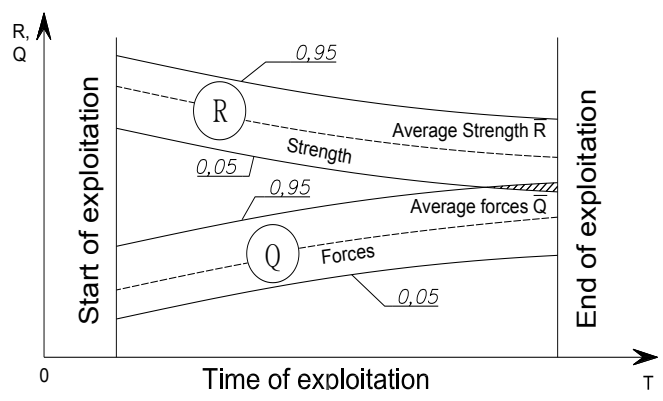

Fig.1. Model of change of resistance R and force of element Q during operation.

This approach opens up the possibility of designing reinforced concrete constructions for a given time of service. This method is particularly useful for constructive calculations of reinforced concrete constructions in cases where the use of standard stochastic methods would be too complicated. Based on the solution of equation (7), the reliability of reinforced concrete structures can be taken as follows:

$$
H=\exp \left\{\frac{T \sigma_{S}}{2 \pi \sqrt{\sigma_{S}^{2}+\sigma_{R}^{2}}} \exp \left[-\frac{\left(m_{R}-m_{S}\right)^{2}}{2\left(\sigma_{S}^{2}+\sigma_{R}^{2}\right)}\right]\right\}
$$

where: $\sigma_{S}^{2}$ - stress dispersion - in the case of solving nonlinear problems, can be determined by the results of the iterative process; $\sigma_{R}^{2}$ - dispersion of bearing capacity; $m_{R}$ is the mathematical expectation of the carrying capacity; $m_{S}$ is the expected stress; $T$ - service life.

If the parameters of the cross section, the type of loading and the static scheme of the construction, as well as the characteristics of the corrosion process are known, then:

$$
H=\exp \left\{\frac{T \sqrt{\alpha^{2}+\beta^{2}}}{2 \pi \sqrt{K^{2} \sigma_{Q}^{2}+K_{c}^{2} \sigma_{R}^{2}}} \exp \left[-\frac{\left(K_{c} m_{R}-K \sigma_{Q}\right)^{2}}{2\left(K^{2} \sigma_{Q}^{2}+K_{c}^{2} \sigma_{R}^{2}\right)}\right]\right\}
$$

where: $\sigma_{Q}$ is the dispersion of the loading effect; $m_{R}$ is the mathematical expectation of the carrying capacity; $K$ - coefficient depending on the size of the cross section of the element of the considered construction; $K_{C}$ is the coefficient taking into account corrosion damage to the construction, $\alpha$ and $\beta$ are the constants of the correlation function, chosen in such a way that the experimental strain curve coincides with the theoretical one.

From where it is possible to easily find the lifetime of the structure, when the parameters of the cross section are known under the condition that $\sigma_{R}=0$ considering the level for which emissions are prohibited (deterministic level):

$$
T=\frac{2 \pi \ln H}{K \sigma_{Q} \exp \left[-\frac{\left(K_{C} m_{R}-K \sigma_{Q}\right)^{2}}{2 K^{2} \sigma_{Q}^{2}}\right]}
$$

Or, if the period of exploitation is known, the cross-sectional dimensions: 


$$
K=\frac{K_{c} m_{R}}{m_{Q}+\sigma_{Q} \sqrt{2 A}}
$$

here:

$$
A=-\ln \frac{2 \pi(H)}{T \sqrt{\alpha^{2}+\beta^{2}}}
$$

The reliability condition that takes into account the statistical variation in loads (forces) and the resistance of materials at time $T=0$ when the density of the load distribution and resistance distant from each other, and the probability of failure at first is small. Over time, the distributions approach each other, forming an intersecting area of increasing size, which illustrates the probability of destruction (Fig.1).

\section{Conclusions}

The safety and operational suitability of buildings and structures are ensured by the perfection of design standards, quality of the project, materials, manufacturing, installation and erection, and operating conditions. Therefore, when designing and calculating, estimates are necessary: on the one hand, the actual bearing capacity and residual durability of existing reinforced concrete constructions that were damaged, on the other hand, the service life of new constructions under development using mathematical models of their wear.

It justifies the minimum permissible level of safety, which guarantees the basic safety of the structure against mechanical stress, taking into account the degradation of the material during operation under the influence of the environment. That is, safety in general includes safety under mechanical stress and safety with respect to the durability of the construction.

In the accepted practice of design, safety under mechanical stress is expressed in terms of material safety factors and loads established by probabilistic methods for the required level of safety. Then durability safety is taken into account by increasing the bearing capacity or other indicators of structural reliability at the beginning of operation so that by its completion the safety level is not exceeded the minimum acceptable value with the required probability. Practically, the safety of the service life is determined by the value of the coefficient of safe service life. It is also assumed that the resistance of the structure $\mathrm{R}$ and the load $Q$ can be given by their characteristic values.

A technique is proposed for a direct probabilistic approach to the assessment of the durability of reinforced concrete constructions, based on the application of the normal distribution law and a priori statistical parameters of resistance, effort and wear rate. The probability of destruction with the simultaneous action of power and non-force factors is determined using the safety characteristic.

The design of the durability of reinforced concrete constructions in the format of the limit state method can be performed by separate or combined calculation methods. In the combined calculation method, the calculation procedure is such that first the calculation is performed on the limiting states, taking into account the results of calculating the durability and the required safety at the end of the service life. This technique is convenient in the case of degradation mechanisms associated with corrosion, which directly affect the bearing capacity or other parameters of reinforced concrete constructions. 
The basic starting points and hypotheses for constructing a computational model of the study are formulated, taking into account the peculiarities of power and non-force imperfections of materials as a result of the manifestation of corrosion damage.

The proposed durability prediction is performed in a deterministic form, which allows to realize calculations at the application level in the format of the limit state method, while maintaining effective safety control during the service life.

The influence of some design structural parameters on the durability of reinforced concrete structures is investigated.

So, the arguments carried out above, lead to the conclusion, that the existing methods for calculating the building constructions of buildings and structures, taking into account durability in conditions of corrosion damage, are still very far from perfect, since they have some, sometimes significant, flaws. Thus, the construction of a methodology for calculating the durability of reinforced concrete constructions with regard to corrosion damage can be a very urgent task, provided it is based on the correct choice of physical, design and mathematical models that most fully take into account the actual work of materials and especially its specificity under the influence of loads of various types.

\section{References}

1. S.V. Aleksandrovsky, Durability of external walling (2004)

2. V.M. Bondarenko, Concrete and reinforced concrete 2, 56-61 (2008)

3. E.M. Zverjaev, M.V. Berlinov, M.N. Berlinova, International Journal of Applied Engineering Research 11-8, 5811 (2016)

4. E.A. Korol, M.V. Berlinov, M.N. Berlinova, Procedia Engineering 153, 292-297 (2016)

5. N.V. Klyueva, Concrete and reinforced concrete 3, 22 (2008)

6. A.G. Tamrazyan, Messenger MGSU 10, 109-115 (2012) 